\title{
A Note on Dynasties and Reigns
}

MAJOR CHINESE DYNASTIES

Zhou (ca. I045-256 B.C.E.)

Qin (22I-206 B.C.E.)

Han (2O2 B.C.E.-C.E. 220)

Tang (6I8-906)

Song (960-I 279)

Yuan (I 279-I368)

Ming (I 368-I 644)

Qing (I644-I9II)

REIGNS OF THE QING DYNASTY AND THEIR ABBREVIATIONS

Shunzhi SZ ( I 644-62)

Kangxi KX (1662-I722)

Yongzheng YZ (1723-36)

Qianlong QL (I736-96)

Jiaqing JQ ( I 796-I 820)

Daoguang DG (I820-5I)

Xianfeng XF (I 85 I-6I)

Tongzhi TZ ( I 86I-75)

Guangxu GX (I875-1908)

Xuantong XT (I908-I2) 
This page intentionally left blank 